\title{
CHILDHOOD BLINDNESS IN INDIA: CAUSES IN 1318 BLIND SCHOOL STUDENTS IN NINE STATES
}

\author{
J. S. RAHI ${ }^{1}$, S. SRIPATHI ${ }^{2}$, C. E. GILBERT ${ }^{1}$ and A. FOSTER ${ }^{1}$ \\ London and Madurai, India
}

\begin{abstract}
SUMMARY
It is estimated that at least 200000 children in India have severe visual impairment or blindness and approximately 15000 are in schools for the blind. Although this represents a small percentage of the estimated 5 million blind in India, it is significant in terms of 'blind-years'. Strategies to combat childhood blindness require accurate data on the causes to allocate resources to appropriate preventive and curative services. Since socio-economic factors vary in different areas of this industrialising country data should be representative of the country as a whole. This is the first multi-state study to be undertaken in India using the Record for Children with Blindness and Low Vision from the World Health Organization/PBL Programme. A total of 1411 children in 22 schools from nine states in different geographical zones were examined by an ophthalmologist and optometrist. Of these, 1318 children were severely visually impaired or blind (SVI/BL). The major causes of SVI/BL in this study were: (1) corneal staphyloma, scar and phthisis bulbi (mainly attributable to vitamin $A$ deficiency) in 26.4\%; (2) microphthalmos, anophthalmos and coloboma in $20.7 \%$; (3) retinal dystrophies and albinism in $19.3 \%$; and (4) cataract, uncorrected aphakia and amblyopia in $12.3 \%$. This mixed pattern of causes lies in an intermediate position between the patterns seen in developing countries and those seen in industrialised countries. The causes identified indicate the importance both of preventive public health strategies and of specialist paediatric ophthalmic and optical services in the management of childhood blindness in India.
\end{abstract}

The problem of childhood blindness is greatest in the developing nations of Asia and Africa where the prevalence of blindness in children (under 16 years)

From: ${ }^{1}$ International Centre for Eye Health, Department of Preventive Ophthalmology, Institute of Ophthalmology, Bath Street, London EC1V 9EL, UK; ${ }^{2}$ Aravind Eye Hospital, Anna Nagar, Madurai, Tamil Nadu, India.

Correspondence to: Allen Foster, FRCS, FRCOphth, Senior Lecturer in Preventive Ophthalmology, Institute of Ophthalmology, Bath Street, London EC1V 9EL, UK. is estimated to be 5 times as high as in the United Kingdom. ${ }^{1}$ Severe visual loss in childhood adversely affects mobility, education and employment opportunities. These have far-reaching implications for the quality of life of children in developing countries. The global estimate of 1.5 million severely visually impaired and blind (SVI/BL) children is relatively small compared with the 17 million adults blind through cataract, but the burden of disability in terms of blind-years is comparable, at approximately 75 million blind-years, because of the duration of life with the visual disability. ${ }^{2}$

The 1991 census of India estimated the population of India to be 850 million, of which 314 million (37\%) are individuals under 16 years of age. ${ }^{3}$ Data on childhood blindness in India are incomplete, but applying an estimated prevalence of $0.7( \pm 0.3)$ per 1000 children to the under- 16 population ${ }^{1}$ provides an estimate of $218000( \pm 92000)$ blind children.

Strategies to combat childhood blindness require accurate, standardised data on the causes. India has a system of federal government and this is reflected in health care and in the socio-economic conditions experienced in different parts of the country. Health policy planning and implementation occur at central and state level. Information on the causes of childhood blindness needs to identify the important causes by state in order to target limited resources to the most important priorities.

The aims of this study were: first, to provide baseline data on the major causes of SVI/BL in children using the WHO/PBL standard reporting form, ${ }^{4}$ second, to identify the avoidable, i.e. readily preventable or treatable, causes of SVI/BL; and third, to describe the variations by state for different causes of childhood blindness in India.

\section{METHODS}

A cross-sectional study design was used to determine the causes of severe visual impairment and blindness in children attending schools for the blind. 
Restriction of the study to these children introduces certain biases which are discussed later. However, the information obtained from blind school studies can provide indications of the relative importance of the different causes of SVI/BL in childhood. This methodology has been used in other developing countries for this purpose.

It is estimated that 15000 children attend residential schools for the blind in India and a further 5000 are in integrated education programmes. ${ }^{5}$ A total of 1411 children attending 22 schools for the blind in nine states of India were examined. This represents approximately $10 \%$ of the residential blind school population. Schools were identified from the Resource Directory of the International Council for the Education of the Visually Handicapped ${ }^{6}$ and through discussion with liaising ophthalmologists in India. For some states of the country no schools could be identified. The selection of schools was non-random. The objective was to examine in each state a minimum of 100 children of both sexes, between the ages of 3 and 15 years, from urban and rural communities. Time and travel constraints to some states imposed limitations on study size and coverage.

The 22 schools in nine states of India were visited in a total period of 12 weeks between mid-March and mid-August 1993. Each school principal was sent an outline of the purpose of the study with a written request for permission to visit the school and carry out ophthalmic examinations of all children under 16 years. All schools approached agreed to inclusion in the study. The senior ophthalmologists at the large ophthalmic units closest to each school were identified and contacted to request support and assistance with the study.

All the children were examined by an ophthalmologist (J.R.) and an optometrist (S.S.). Each child was seen with his or her class teacher and parent, whenever possible. Information was gathered by interview and by consulting medical records held with school registration documents. Socio-demographic data of age, sex, area of usual residence, place of birth, ethnic group and paternal occupation or caste were recorded. A routine medical history was taken and relevant details of family history and the mother's obstetric history were noted.

Each child was assessed by the optometrist. Uniocular and binocular visual acuity measurements, with correction if usually worn, were made using an Illiterate $\mathrm{E}$ chart. These were recorded according to the WHO categories of classification ${ }^{7}$ (Table I). Functional vision, such as the ability to walk around unaided or to recognise faces, was assessed. Visual fields were routinely assessed by confrontation. Refraction, after cycloplegia, was carried out unless it was inappropriate (e.g. in children with bilateral phthisis bulbi). Anterior segment examination was undertaken by the ophthalmologist using a magnifying loupe and torch. Posterior segment examination was by direct and/or indirect ophthalmoscopy after pupillary dilation. Intraocular pressures were not routinely measured.

All personal data and clinical findings for each child were recorded on the WHO/PBL Eye Examination Record for Children with Blindness and Low Vision in accordance with the coding instructions. ${ }^{4}$ For each child the causes of visual loss were recorded using the anatomical and aetiological (based on time of insult resulting in visual deficit) classification used in the form.

The findings of the study relating to the major anatomical and aetiological causes of SVI/BL are presented.

\section{RESULTS}

In total 1411 children between the ages of 3 and 15 years were examined. Of these, $1318(93 \%)$ were SVI/BL and the results presented here relate to these children (Table I).

Fifty-eight per cent (764) of the SVI/BL children were male. Four per cent (53) of the SVI/BL children were under 5 years, $40 \%$ (527) between 6 and 10 years and $56 \%$ (738) between 11 and 15 years of age.

\section{Anatomical Site of Abnormality}

The anatomical classification of causes of SVI/BL is presented in Table II.

Corneal diseases (including those leading to phthisis bulbi following corneal perforation) accounted for $26.4 \%$ (348) of all children with SVI/ $\mathrm{BL}$ : within this group vitamin A deficiency (VAD) was responsible in $245(18.6 \%)$ children, measles in $42(3.2 \%)$, ophthalmia neonatorum in $10(0.8 \%)$, and other causes of corneal scarring such as trauma, keratitis and harmful traditional eye medicines in 45 $(3.4 \%)$ children.

Congenital anomalies of the whole globe, i.e. anophthalmos (72) and microphthalmos (156), were responsible for $17.3 \%$ of cases of SVI/BL. Unknown causes affecting the whole globe - phthisis bulbi (50), disorganised globe (53) and removed globes (3) accounted for a further $8.0 \%$ of SVI/BL.

Retinal disorders were responsible for $20.7 \%$ (273) of children with SVI/BL. The majority were retinal

Table I. Visual acuity in the better eye in 1411 children in blind schools in India

\begin{tabular}{lcrr}
\hline WHO category & Level of vision & \multicolumn{1}{c}{$n$} & \multicolumn{1}{c}{$\%$} \\
\hline No visual impairment & $6 / 18$ or better & 12 & 0.9 \\
Visual impairment & $<6 / 18-6 / 60$ & 81 & 5.7 \\
Severe visual impairment & $<6 / 60-3 / 60$ & 113 & 8.0 \\
Blind & $<3 / 30-$ NPL & 1205 & 85.4 \\
Total & & 1411 & 100.0 \\
\hline
\end{tabular}


Table II. Anatomical classification of the causes of SVI/BL in 1318 blind school children in India

\begin{tabular}{lcc}
\hline Anatomical site & $n$ & $\%$ \\
\hline Whole glibbe $^{\mathrm{a}}$ & 334 & 25.3 \\
Cornea $^{\mathrm{b}}$ & 348 & 26.4 \\
Lens & 162 & 12.3 \\
Retina & 273 & 20.7 \\
Uvea & 77 & 5.8 \\
Optic nerve & 78 & 5.9 \\
Buphthalmos/glaucoma & 34 & 2.6 \\
Others & 12 & 0.9 \\
Total & 1318 & 100.0 \\
\hline
\end{tabular}

${ }^{a}$ Includes anophthalmos/microphthalmos/phthisis of unknown aetiology.

${ }^{\mathrm{b}}$ Includes phthisis following diseases of the cornea.

dystrophies (242) and albinism (13), with other retinal disorders including retinopathy of prematurity (3) accounting for the remainder.

Disorders of the lens were seen in $12.3 \%$ (162) of children. Sixty-three had unoperated cataract, 67 children were aphakic and/or amblyopic and the remaining 32 children had visual loss from postoperative complications.

Disorders of the uvea were seen in $5.8 \%$ of children: coloboma (46), aniridia (16) and uveitis (15).

Optic nerve disorders were seen in $5.9 \%$ of children, secondary optic atrophy (59) and optic nerve hypoplasia (14) being the major causes.

Buphthalmos and glaucoma were diagnosed in $2.6 \%(34)$ of SVI/BL children.

High refractive errors (8) and cortical blindness (4) accounted for the remaining $1 \%$ of SVI/BL children.

\section{Aetiology}

The causes of SVI/BL are classified according to the time of known insult resulting in visual loss in Table III.

Prenatal factors, i.e. hereditary or intra-uterine, were definitely identified in $25 \%$ of children. In $28 \%$ of children postnatal factors acquired in infancy or childhood were the cause of visual loss. Perinatal factors were identified in only $1 \%$ and in the remaining $46 \%$ the aetiology was undetermined. In
Table III. Causes of SVI/BL in 1318 children in India by aetiological category

\begin{tabular}{lrr}
\hline Aetiological category $^{\mathrm{a}}$ & $n$ & $\%$ \\
\hline Hereditary & 303 & 22.9 \\
Intra-uterine & 23 & 1.8 \\
Perinatal & 18 & 1.4 \\
Childhood & 367 & 27.9 \\
Undetermined & 288 & 21.8 \\
$\quad$ Present at birth & 319 & 24.2 \\
$\quad$ Unknown onset & 1318 & 100.0 \\
Total & &
\end{tabular}

${ }^{\mathrm{a}}$ Categorisation according to time of insult leading to visual loss.

this undetermined group, 288 children had congenital ocular anomalies of prenatal origin which could not, however, be definitely identified as hereditary or as due to factors operating during the intra-uterine period. When these children are included with those in whom a definite hereditary or intra-uterine factor was identified, $47 \%$ of cases of SVI/BL are attributable to prenatal factors.

\section{Avoidable Causes}

In Table IV the causes which are either readily preventable or treatable have been summarised. Overall $622(47 \%)$ children had avoidable causes of SVI/BL: $31 \%$ had preventable causes and 16\% treatable causes.

\section{Variation in Major Causes by State}

Variation in the relative importance of each of the major causes of SVI/BL was observed between states (Table V).

Vitamin A deficiency was proportionately more important in Madhya Pradesh $(27 \%)$ and West Bengal $(25 \%)$ but relatively unimportant in Karnataka (12\%) and Kerala (8\%).

Retinal dystrophies and albinism were relatively more important in Kerala (33\%) and Haryana (26\%) and relatively less important in Uttar Pradesh (7\%) and Gujarat (9\%).

Anomalies of the globe were responsible for 33\% SVI/BL in Uttar Pradesh, 29\% in Karnataka and 6\% in Kerala.

Table IV. Causes of avoidable childhood SVI/BL in 1318 blind school children in India

\begin{tabular}{|c|c|c|c|c|c|}
\hline Preventable & $n$ & $\%$ & Treatable & $n$ & $\%$ \\
\hline $\begin{array}{l}\text { VAD } \\
\text { Measles } \\
\text { HTEM }^{\mathrm{a}} \\
\text { Meningitis }_{\text {Trauma }} \\
\text { Rubella } \\
\text { Toxoplasmosis } \\
\text { Ophth. neonatorum } \\
\text { Others (e.g. drugs) }\end{array}$ & $\begin{array}{r}245 \\
42 \\
11 \\
53 \\
6 \\
9 \\
8 \\
10 \\
24\end{array}$ & $\begin{array}{r}18.6 \\
3.2 \\
0.8 \\
4.0 \\
0.5 \\
0.7 \\
0.6 \\
0.8 \\
1.8\end{array}$ & $\begin{array}{l}\text { Cataract/aphakia } \\
\text { Buphth/glaucoma } \\
\text { Ocular trauma } \\
\text { ROP }^{c}\end{array}$ & $\begin{array}{r}162 \\
34 \\
15 \\
3\end{array}$ & $\begin{array}{r}12.3 \\
2.8 \\
1.1 \\
0.2\end{array}$ \\
\hline Sub-total & 408 & 31.0 & & 214 & 16.3 \\
\hline
\end{tabular}

${ }^{\mathrm{a}}$ Harmful traditional eye medicines.

${ }^{\mathrm{b}}$ Head trauma.

${ }^{\mathrm{c}}$ Retinopathy of prematurity. 
Table V. Major causes of SVI/BL in 1318 children in 9 states of India

\begin{tabular}{|c|c|c|c|c|c|c|c|c|}
\hline \multirow[t]{2}{*}{ State } & \multicolumn{2}{|c|}{$\mathrm{VAD}^{\mathrm{a}}$} & \multicolumn{2}{|c|}{ Ret. dys./Al. ${ }^{b}$} & \multicolumn{2}{|c|}{ Cat./Aph. $^{\mathrm{c}}$} & \multicolumn{2}{|c|}{ An./Mi./Col. ${ }^{\mathrm{d}}$} \\
\hline & $n$ & $\%$ & $n$ & $\%$ & $n$ & $\%$ & $n$ & $\%$ \\
\hline $\begin{array}{l}\text { Gujarat } \\
(n=97)\end{array}$ & 21 & 21.6 & 9 & 9.2 & 11 & 11.3 & 17 & 17.5 \\
\hline $\begin{array}{l}\text { Madhya Pradesh } \\
(n=101)\end{array}$ & 27 & 26.7 & 12 & 11.9 & 4 & 3.9 & 19 & 18.8 \\
\hline $\begin{array}{l}\text { Haryana } \\
(n=141)\end{array}$ & 22 & 15.6 & 36 & 25.5 & 8 & 5.7 & 29 & 20.6 \\
\hline $\begin{array}{l}\text { Uttar Pradesh } \\
(n=134)\end{array}$ & 29 & 21.6 & 9 & 6.7 & 4 & 2.9 & 44 & 32.8 \\
\hline $\begin{array}{l}\text { West Bengal } \\
(n=89)\end{array}$ & 22 & 24.7 & 10 & 11.2 & 15 & 16.8 & 17 & 19.1 \\
\hline $\begin{array}{l}\text { Maharashtra } \\
(n=157)\end{array}$ & 32 & 20.4 & 22 & 13.8 & 20 & 12.7 & 27 & 17.2 \\
\hline $\begin{array}{l}\text { Karnataka } \\
(n=122)\end{array}$ & 14 & 11.5 & 29 & 23.7 & 8 & 6.5 & 35 & 28.7 \\
\hline $\begin{array}{l}\text { Kerala } \\
(n=93)\end{array}$ & 7 & 7.5 & 30 & 33.2 & 22 & 23.6 & 6 & 6.5 \\
\hline $\begin{array}{l}\text { Tamil Nadu } \\
(n=384)\end{array}$ & 71 & 18.5 & 98 & 25.5 & 38 & 9.8 & 79 & 20.6 \\
\hline
\end{tabular}

$n=$ Number of children with SVI/BL.

${ }^{\mathrm{a}}$ Vitamin A deficiency.

${ }^{\mathrm{b}}$ Retinal dystrophies and albinism.

${ }^{c}$ Cataract and aphakia/amblyopia.

${ }^{\mathrm{d} A n o p h t h a l m o s, ~ m i c r o p h t h a l m o s ~ a n d ~ c o l o b o m a . ~}$

Cataract was responsible for $24 \%$ SVI/BL in Kerala, $4 \%$ in Madhya Pradesh and 3\% in Uttar Pradesh.

\section{DISCUSSION}

There is currently no system of blindness registration in India, so a community-based prevalence study would be required to provide accurate cause-specific prevalence data. The estimated all-cause blindness prevalence of 0.7 per 1000 children $^{1}$ would dictate a very large study. Small studies of well-defined communities must be interpreted with caution in a country as varied as India. The present study of children in schools for the blind cannot provide cause-specific prevalence rates and it is likely to under-represent certain groups of visually handicapped children, i.e. those who have limited access to schools for the blind because of lack of parental awareness or distance of residence from school; preschool age children; those with multiple disabilities; and those with unilateral or mild bilateral visual impairment. Children in this study are, therefore, likely to represent those Indian children in whom $\mathrm{SVI} / \mathrm{BL}$ is the main disability and who gain admission to a school for the blind.

Blindness from VAD is known to be associated with a particularly high mortality ${ }^{8}$ and affected children may die before reaching school age and therefore be unrepresented in this study. However, VAD was identified as the most common single cause of SVI/BL in this study and, taken together with measles, accounted for $22 \%$ of cases. The relative importance of VAD in each state varied from $8 \%$ in Kerala to $27 \%$ in Madhya Pradesh. These findings need to be interpreted with caution and require further validation to determine the prevalence of VAD and associated risk factors by state. The data presented on SVI/BL due to VAD can be used to indicate those states which are most likely to have the highest prevalence rates of VAD.

Similar studies in Africa have shown that VAD accounts for $50-70 \%$ of SVI/BL in childhood in East Africa $^{9}$ and $26 \%$ in West Africa. ${ }^{10}$ There is a varied and complex interaction of factors which result in visual loss from corneal scarring due to VAD and measles, emphasising the need for local evaluation of the situation once corneal scarring from VAD/ measles is identified as an important cause of visual loss in children.

Overall $23 \%$ of children had SVI/BL definitely attributable to genetic disease. The mode of inheritance in the majority of these children was autosomal recessive. Retinal dystrophies (Leber's amaurosis, retinitis pigmentosa and achromatopsia) and albinism accounted for $19 \%$ of cases. Cataract and optic nerve disease were also important and accounted for approximately half of the autosomally dominant inherited diseases. Consanguineous marriages, which increase the likelihood of autosomal recessive diseases, are common in certain communities in India. First-cousin marriages occur throughout the country and maternal uncle/niece marriages are common in South India. Retinal dystrophies and albinism accounted for $33 \%$ and $26 \%$ of all SVI/BL cases in the southern states of Kerala and Tamil Nadu.

Congenital ocular anomalies (anophthalmos, microphthalmos, coloboma and aniridia) accounted for $22 \%$ of children with SVI/BL. These conditions appear to be relatively more important in India than 
in other developing countries, The highest combined prevalence of microphthalmos and coloboma reported in blind school surveys is $11.2 \%$ in Japan. ${ }^{11}$ Known causes of these congenital anomalies include chromosomal and genetic disorders, viral infections during pregnancy and certain teratogenic drugs. ${ }^{12}$ In animal models, deficiencies of vitamin A, vitamin $B$ complex and trace elements ${ }^{13}$ are all associated with these anomalies. The reasons for the high proportion of cases of anophthalmos and microphthalmos in this study are unknown.

Twelve per cent of children had SVI/BL from disorders of the lens. Two-fifths of these children were aphakic and/or amblyopic as a result of late surgery or inadequate refractive correction. A further two-fifths had unoperated cataracts and the remainder had complications following cataract surgery. These findings indicate the need for specialist paediatric surgical and optical services for detection and treatment of cataract in infancy to avoid severe visual loss from cataract and amblyopia.

Other causes of SVI/BL requiring specialist ophthalmic interventions were buphthalmos/glaucoma $(3 \%)$ and some corneal scars amenable to treatment.

Causes which are potentially preventable include $\mathrm{VAD}$, measles, congenital rubella, congenital toxoplasmosis and optic atrophy following meningitis. Improved nutrition, immunisation and early treatment of systemic infections could reduce childhood blindness from these causes.

The pattern seen in this blind school study in India is a 'mixed' intermediate pattern. It is dissimilar to the pattern seen in the developing countries of Africa $^{14}$ (where corneal scarring from VAD and measles accounts for up to $70 \%$ of childhood SVI/ $\mathrm{BL}$ ) and dissimilar to that seen in the industrialised countries of Europe ${ }^{15}$ and North America ${ }^{16}$ (where up to $40 \%$ of severe visual loss is due to retinal disease). In this study the proportions of children with SVI/BL due to corneal scarring, retinal disease and lesions of the whole globe were similar, being approximately $20-25 \%$ each.

The importance of hereditary factors $(23 \%)$ and acquired childhood factors $(28 \%)$ contrasts with the small contribution from perinatal and intra-uterine factors. However, this study may underestimate the importance of both genetic and intra-uterine factors since in $22 \%$ of cases the abnormality had been present at birth but was of undetermined aetiology. In industrialised countries hereditary and perinatal factors predominate, while in Africa childhood factors predominate. In this study, prenatal factors were responsible for at least $47 \%$ of $S V I / B L$ and this identifies an important area for future research.

The variations observed between states reflect, in part, the evolving socio-economic conditions experi- enced in the industrialisation of India. Further studies will be needed to quantify the changing patterns of childhood blindness with time in individual states.

\section{CONCLUSION}

In common with other developing countries, a large proportion of the childhood blindness in India is avoidable. Action is needed to prevent blindness from vitamin A deficiency, measles and external ocular infections, particularly in those states where corneal scarring accounts for more than $10 \%$ of childhood blindness.

Specialist paediatric and optical services are required to manage cataract and glaucoma in childhood and to provide assessment and services for children with low vision.

Further work is indicated to elucidate the causes of anophthalmos, microphthalmos and coloboma which together account for 1 in 6 cases. It would also be useful to document the changing patterns in the causes of childhood blindness in individual states to allow early action against emerging avoidable causes.

The authors acknowledge financial help from CBM and the Tijssen Foundation.

Key words: Albinism, Anophthalmos, Blindness, Cataract, Childhood, Coloboma, India, Microphthalmos, Multi-state, Severe visual impairment (SVI), Retinal dystrophies, Vitamin A deficiency, WHO/PBL Eye Examination Record.

\section{REFERENCES}

1. Foster A, Gilbert CE. Epidemiology of childhood blindness. Eye 1992;6:173-6.

2. Foster A. Worldwide blindness, increasing but avoidable. Semin Ophthalmol 1993;8:166-70.

3. World Development Report 'Investing in Health'. World Bank/Oxford University Press, 1993.

4. Gilbert CE, Foster A, Thylefors B, Negrel DA. Childhood blindness: a new form for recording the causes of visual loss in children. WHO Bull 1993;71:485-9.

5. Michael PG, CBM Asia. Personal communication, 1993.

6. Resource Directory of the International Council for the Education of the Visually Handicapped. Bensheim, Germany, 1986.

7. Adaptation of the International Classification of Diseases, 1975 revision. Geneva: WHO, 1977.

8. Report of the WHO Meeting on Prevention of Childhood Blindness. WHO/Prevention of Blindness Programme, 1990. Unpublished.

9. Foster A, Sommer A. Childhood blindness from corneal ulceration in Africa: causes, prevention and treatment. WHO Bull 1986;64:619-23.

10. Gilbert CE, Canovas R, Hogan, Rao S, Foster A. Causes of childhood blindness: results from West Africa, South India and Chile. Eye 1993;7:184-8.

11. Fujiki K, Nakajima A. Yasuda N, Tanabe U, Kabasawa K. Genetic analysis of microphthalmos. Ophthalmic Paediatr Genet 1982;1:139-49. 
12. Warburg M. An update on microphthalmos and coloboma. Ophthalmic Paediatr Genet 1991;12:57-63.

13. McLaren DS. Malnutrition and the eye. New York: Academic Press, 1963.

14. Foster A. Causes of world blindness. Community Eye Health 1988;1:2-3.

15. Goggin M, O'Keefe M. Childhood blindness in the
Republic of Ireland: a national survey. $\mathrm{Br} \mathrm{J}$ Ophthalmol 1991;75:425-9.

16. Khan HA, Moorhead HB. Statistics on blindness in the Model Reporting Area 1969-1970. US Department of Health, Education and Welfare Publication no. 73-427. Washington, DC: US Government Printing Office, 1973. 